\title{
Corf-94/129--26
}

UCRL-JC-117697

PREPRINT

\section{Spectral Measurements of Few-Electron Uranium Ions Produced and Trapped in a High-energy Electron Beam Ion Trap}

\author{
P. Beiersdorfer
}

Prepared for Submittal to

Nuclear Instruments \& Methods

13th International Conference on the

Application of Accelerators in Research and Industry

Denton, Texas, Nov. 7-10, 1994

November 4, 1994

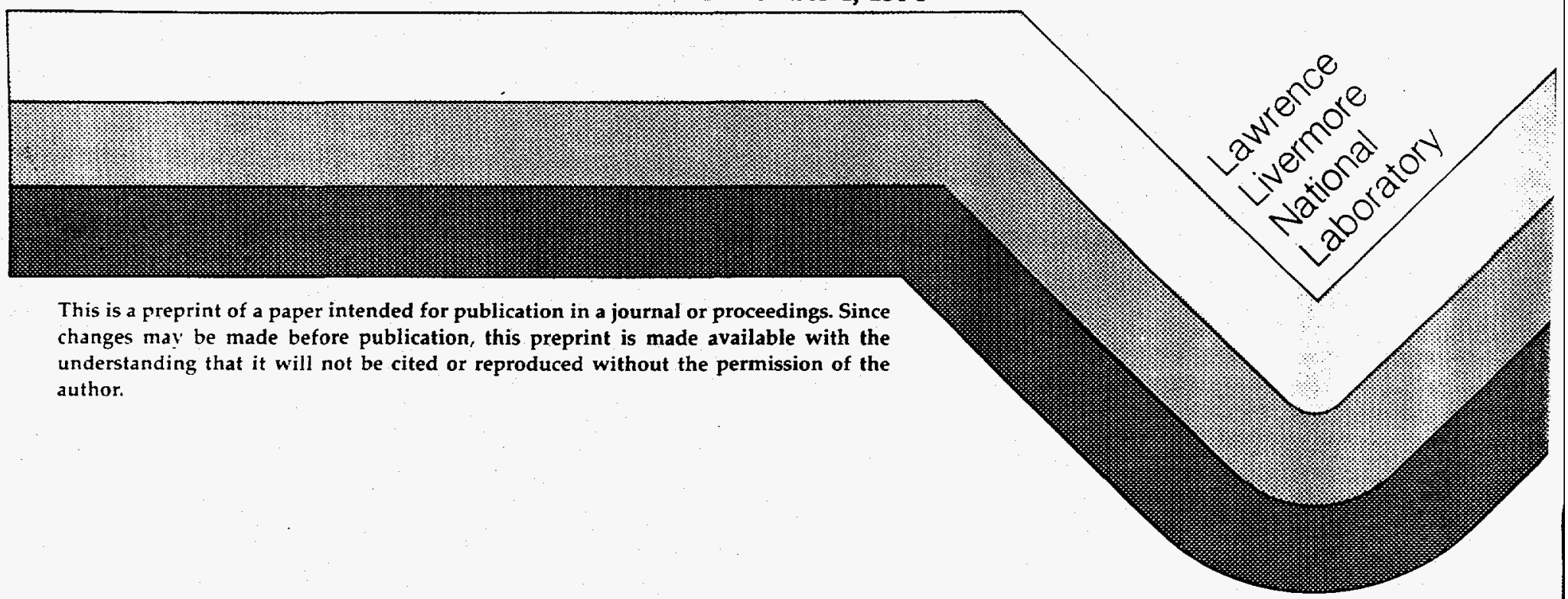

This is a preprint of a paper intended for publication in a journal or proceedings. Since changes may be made before publication, this preprint is made available with the understanding that it will not be cited or reproduced without the permission of the uthor. 


\section{DISCLAIMER}

This document was prepared as an account of work sponsored by an agency of the United States Government. Neither the United States Government nor the University of California nor any of their employees, makes any warranty, express or implied. or assumes any legal liability or responsibility for the accuracy: completeness. or usefulness of any information, apparatus, product, or process disclosed. or represents that its use would not infringe privately owned rights. Reference herein to any specific commercial products, precess, or service by trade name. trademark, manufacturer, or otherwise, does not necessarily constitute or imply its endorsement. recommendation. or favoring by the United States Government or the University of California. The views and opinions of authors expressed herein do not necessarily state or reflect those of the Linited States Government or the Liniversity of California, and shall not be used for advertising or product endorsement purposes. 


\section{DISCLAIMER}

Portions of this document may be illegible in electronic image products. Images are produced from the best available original document. 


\title{
Spectral measurements of few-electron uranium ions produced and trapped in a high-energy electron beam ion trap
}

\author{
P. Beiersdorfer \\ Department of Physics and Space. Technology \\ Lawrence Livermore National Laboratory \\ Livermore, CA 94551
}

\begin{abstract}
Measurements of $2 s_{1 / 2}-2 p_{3 / 2}$ electric dipole and $2 p_{1 / 2}-2 p_{3 / 2}$ magnetic dipole and electric quadrupole transitions in $\mathrm{U}^{82+}$ through $\mathrm{U}^{89+}$ have been made with a high-resolution crystal spectrometer that recorded the line radition from stationary ions produced and trapped in a high-energy electron beam ion trap. From the measurements we infer $-39.21 \pm 0.23 \mathrm{eV}$ for the QED contribution to the $2 s_{1 / 2}-2 p_{3 / 2}$ transition energy of lithiumlike $U^{89+}$. A comparison between our measurements and various computations illustrates the need for continued improvements in theoretical aproaches for calculating the atomic structure of ions with two or more electrons in the $L$ shell.
\end{abstract}




\section{Introduction}

The strong field of a heavy nucleus provides an ideal testing ground for the non-perturbative contributions from quantum electrodynamics (QED) to the atomic structure, especially for the contributions from the electron self energy. Because the QED contributions scale approximately as $n^{-3}$, where $n$ is the principle quantum number, they are largest for $1 s$ orbitals. For hydrogenlike uranium $U^{91+}$ the contribution is about $460 \mathrm{eV}$. By contrast, the contribution to the $2 s$ orbital in lithiumlike $\mathrm{U}^{89+}$ is about $47 \mathrm{eV}$, and that to the $3 s$ orbital in sodiumlike $\mathrm{U}^{79+}$ is about $11 \mathrm{eV}$. Spectroscopic techniques, however, cannot measure these values directly but are sensitive only to the differences between two energy levels. As a fraction the energy differences, QED effects are largest for $\Delta n=0$ transitions that connect levels in the $n=2$ shell. Here, typical transition energies range from about $300-4500 \mathrm{eV}$, and the $2 s$-QED contributions may represent up to $10 \%$ of the total energy. By contrast, transitions that connect to the $1 s$ orbital have energies near 100 $\mathrm{keV}$, so that the $1 s$-QED contribution represents less than $0.5 \%$ of the total.

Because the fractional QED contribution is largest for $\Delta n=0$ transitions we have concentrated our studies on measuring intrashell transitions connecting levels in the $n=2$ shell. In particular, we focused on the $2 s_{1 / 2}-2 p_{3 / 2}$ and $2 p_{1 / 2}-2 p_{3 / 2}$ transitions, which for uranium ions fall into the wavelength region $3500-4500 \mathrm{eV}$. The measurements of the $2 s_{1 / 2}-2 p_{3 / 2}$ transitions provide an excellent measure of the QED effects, as discussed earlier in Ref. 1. The $2 p_{1 / 2}-2 p_{3 / 2}$ transitions, on the other hand, are much less affected by QED effects, as they do not involve a $2 s$ orbital. As a result, a measurement of these transitions can be used to check the non-QED part of relativistic atomic 
structure theory, which is common to all 2-2 transitions. In the following we present an overview of these measurements and discuss the implications for testing and developing atomic structure theory of very highly charged ions.

\section{Experiment}

The highly charged uranium ions needed for our measurements were produced in the high-energy electron beam ion trap (also dubbed SuperEBIT) at the Lawrence Livermore National Laboratory. The device uses an intense electron beam to electrostatically trap, ionize, and excite the uranium ions. ${ }^{2}$ To obtain spectra corresponding to different ionization balances in the trap the beam energy was set to energies ranging from 100-125 keV using electron beam currents between 70 and $185 \mathrm{~mA} .^{1} \mathrm{~A}$ 3-T magnetic field was employed to squeeze the beam to a $30-\mu \mathrm{m}$ radius, so that the electron density was about $3 \times 10^{12} \mathrm{~cm}^{-3}$.

The $x$-ray line emission was analyzed with a von Hámos-type crystal spectrometer. ${ }^{3}$ The spectrometer used a $120 \times 50 \times 0.25 \mathrm{~mm}^{3} \mathrm{LiF}(200)$ crystal with a lattice spacing $2 d=4.027 \AA$. The crystal was bent to a $30-\mathrm{cm}$ radius of curvature. The diffracted $x$ rays were recorded with a gas-filled proportional counter with a $10 \times 3 \times 0.4 \mathrm{~cm}^{3}$ active volume. The working gas consisted of $70 \%$ xenon and $30 \%$ methane at 1.2 -atm overpressure. The resolving power of the setup was about $\lambda / \Delta \lambda=2200$, and was mainly limited by the $270-\mu \mathrm{m}$ spatial resolving power of the detector.

High-resolution $x$-ray spectra of the $2 s_{1 / 2}-2 p_{3 / 2}$ and $2 p_{1 / 2}-2 p_{3 / 2}$ transitions are shown in Fig. 1 (a) and (b), respectively. Contributions from the charge states lithiumlike $U^{89+}$ through neonlike $U^{82+}$ are seen. A total of 
eleven distinct features have been identified in the $2 s_{1 / 2}-2 p_{3 / 2}$ spectrum and eight features in the $2 p_{1 / 2}-2 p_{3 / 2}$ spectrum. These correspond to thirteen electric dipole, nine magnetic dipole, and one electric quadrupole transition.

The $2 s_{1 / 2}-2 p_{3 / 2}$ spectrum was calibrated using the Ly- $\beta, \mathrm{Ly}-\gamma$, and Ly- $\delta$ transitions in hydrogenlike potassium, as described in Ref. 1 ; the $2 p_{1 / 2}-2 p_{3 / 2}$ spectrum was calibrated using the corresponding transitions in hydrogenlike argon. The lines of hydrogenlike ions represent a good choice for calibration because their energies are theoretically well known. Hydrogenic systems are the simplest atomic systems, and both the Dirac and QED energies can be calculated with the highest precision. ${ }^{4-6}$

\section{Results and Comparison with Theory}

The energy of the $2 s_{1 / 2}-2 p_{3 / 2}$ transitions in lithiumlike $\mathrm{U}^{89+}$ was measured to be $4459.37 \mathrm{eV} .^{1}$ Adding the uncertainties arising from the detector nonlinearity, the uncertainty in the calibration lines, and the uncertainty in the dispersion in quadrature with the statistical uncertainty of the position of the measured lithiumlike uranium line we find an overall uncertainty of $\pm 0.21 \mathrm{eV}$ at the $68-\%$ confidence limit, as summarized in Table I. The non-QED part of the transition energy was calculated by Blundell, Johnson, and Sapirstein with many-body perturbation theory (MBPT) to amount to $4498.58 \pm 0.09$ $\mathrm{eV}{ }^{7}$ The QED contribution can thus be isolated from the measured transition energy by subtracting the MBPT value, and we find $-39.21 \pm 0.23 \mathrm{eV}$. We are not aware of a direct calculation of the QED contribution. However, we can estimated it by noting that the calculated QED contribution to the $2 s_{1 / 2}-2 p_{1 / 2}$ splitting is $-41.43 \mathrm{eV},{ }^{8}$ while that for the $2 p_{1 / 2}-2 p_{1 / 2}$ splitting is 
estimated at $2.15 \mathrm{eV}{ }^{7}$ The difference is $-39.28 \mathrm{eV}$. This value is in very good agreement with the QED contribution inferred from our measurement. We note, however, that including the effects of core relaxation, nuclear recoil, and nuclear polarization will change the value of the calculated QED contribution by about $0.1-0.3 \mathrm{eV}$. For example, the effect of nuclear polarization was calculated to shift the $2 s_{1 / 2}-2 p_{1 / 2}$ splitting by about $0.18 \mathrm{eV},{ }^{9}$ while nuclear recoil contributes $-0.08 \mathrm{eV}^{8}$ A definitive comparison with theoretical predictions thus has yet to made.

The ability to perform accurate calculations of the non-QED energies is a prerequisite to isolate the QED contributions from measured transition energies. This ability has existed for several years because of the simplicity of the lithiumlike ion, which has only one valence electron. ${ }^{10}$ In the case of multi-electron ions with more than one valence electron a standard approach for calculating the transition energies is the multi-configuration Dirac-Fock approach (MCDF). ${ }^{11} \mathrm{~A}$ comparison between the measured $2 s_{1 / 2}-2 p_{3 / 2}$ and $2 p_{1 / 2}-2 p_{3 / 2}$ energy splittings and the values calculated with the MCDF approach is given in Fig. 2. The MCDF calculations were performed with the procedure described in Ref. 1. Looking at Fig. 2 we make the following observations. First, the difference between MCDF and measured transition energies is larger for the $2 s_{1 / 2}-2 p_{3 / 2}$ transitions than for the $2 p_{1 / 2}-2 p_{3 / 2}$ transitions. This is because of a $2-\mathrm{eV}$ error ${ }^{1}$ made by the calculations in estimating the QED contribution to the $2 s_{1 / 2}-2 p_{3 / 2}$ transitions by scaling hydrogenic QED values. This error is comparatively insignificant for the $2 p_{1 / 2}-2 p_{3 / 2}$ transitions. Second, the overall difference between calculation and measurement increases as the number of valence electrons increases. For the transitions in carbonlike, nitrogenlike, and oxygenlike uranium the difference is about $2 \mathrm{eV}$ 
(in the case of the $2 s_{1 / 2}-2 p_{3 / 2}$ transitions, the 2-eV error in the QED contribution was subtracted). An accurate test of QED calculations can thus not be performed, as long as there are several $\mathrm{eV}$ uncertainties in the non-QED energies.

Very recently, MBPT was successfully applied to the calculation of the $2 s_{1 / 2}-2 p_{3 / 2}$ transition energies in berylliumlike, carbonlike, fluorinelike, and neonlike uranium. ${ }^{12}$ The calculations were carried out using two different starting potentials in order to check the convergence of the method. A comparison of the results from the two sets of MBPT calculations with our measurements is given in Fig. 2. The agreement with our data is much better than with the MCDF results. However, significant differences still exist, especially for the results for berylliumlike uranium, and no such calculations have yet been performed for boronlike, oxygenlike, and nitrogenlike ions.

\section{Conclusion}

A set of $23 \Delta n=0$ transitions along the isonuclear sequence of very highly charged uranium has been measured with high-resolution crystal spectroscopy. The measurements test the predictions of the QED contributions in the high-Z limit and represent benchmarks for the development of new theoretical techniques for determining the structure of high- $Z$ ions. Experiments are planned to extend these measurements along the isoelectronic sequence.

Moreover, the accuracy of the measurements we have presented are comparable to the effects of nuclear polarization, nuclear recoil, and variations in nuclear size. Measurements are currently under way that have yet higher accuracy and that can distinguish between the transition energies from dif- 
ferent isotopes of the same element. With such measurements we hope to probe and isolate nuclear effects.

\section{Acknowledgment}

Contributions by Mau Chen, Steve Elliott, David Knapp, and Ross Marrs are gratefully acknowledged. This work was performed under the auspices of the U. S. Department of Energy by the Lawrence Livermore National Laboratory under contract W-7405-ENG-48. 


\section{References}

${ }^{1}$ P. Beiersdorfer, D. Knapp, R. E. Marrs, S. R. Elliott, and M. H. Chen, Phys. Rev. Lett. 71, 3939 (1993).

${ }^{2}$ D. A. Knapp, R. E. Marrs, S. R. Elliott, E. W. Magee, and R. Zasadzinski, Nucl. Instrum. Methods A334, 305 (1993).

${ }^{3}$ P. Beiersdorfer, R. E. Marrs, J. R. Henderson, D. A. Knapp, M. A. Levine, D. B. Platt, M. B. Schneider, D. A. Vogel, and K. L. Wong, Rev. Sci. Instrum. 61, 2338 (1990).

${ }^{4}$ J. D. Garcia and J. E. Mack, J. Opt. Soc. Am. 55, 654 (1965).

${ }^{5}$ G. W. Erickson, J. Phys. Chem. Ref. Data 6, 831 (1977).

${ }^{6}$ W. R. Johnson and G. Soff, At. Data Nucl. Data Tables 33, 405 (1985).

${ }^{7}$ S. A. Blundell, W. R. Johnson, and J. Sapirstein, Phys. Rev. A 41, 1698 (1990).

${ }^{8}$ S. A. Blundell, Phys. Rev. A 46, 3762 (1992).

${ }^{9}$ G. Plunien, B. Müller, W. Greiner, and G. Soff, Phys. Rev. A 43, 5853 (1991).

${ }^{10}$ W. R. Johnson, S. A. Blundell, and J. Sapirstein, Phys. Rev. A 37, 2764 (1988).

${ }^{11}$ J. P. Desclaux, in Atomic Theory Workshop on Relativistic and QED Effects in Heavy Atoms, AIP conference Proceedings No. 136, edited by $\mathrm{H}$. P. Kelly and Y.-K. Kim (AIP, New York, 1985). 
${ }^{12}$ W. R. Johnson, J. Sapirstein, and K. T. Cheng, submitted to Phys. Rev. A (1994). 


\section{Tables}

TABLE I. Contributions to the $0.21-\mathrm{eV}$ uncertainty in the measured energy of the $2 \mathrm{~s}_{1 / 2}-2 \mathrm{p}_{3} / 2$ transition in lithiumlike $\mathrm{U}^{89+}$.

\begin{tabular}{ll}
\hline \multicolumn{1}{c}{ Source } & Magnitude \\
\hline Position of uranium line & $0.12 \mathrm{eV}$ \\
Position of calibration lines (dispersion) & $0.08 \mathrm{eV}$ \\
Uncertainty in energies of calibration lines & $0.10 \mathrm{eV}$ \\
Detector linearity & $0.12 \mathrm{eV}$ \\
\hline
\end{tabular}




\section{Figures}

FIG. 1. Spectra of 2-2 transitions in highly charged uranium: (a) $2 s_{1 / 2}-2 p_{3 / 2}$ electric dipole transitions; (b) $2 p_{1 / 2}-2 p_{3 / 2}$ magnetic dipole transitions. Lines are labeled by the charge state of the emitting ion. C(E2) denotes an electric quadrupole transition in carbonlike uranium.

FIG. 2. Differences between measured and theoretical transition energies for different charge states of uranium. The difference with MCDF calculations for the $2 s_{1 / 2}-2 p_{3 / 2}$ and $2 p_{1 / 2}-2 p_{3 / 2}$ transitions are shown as solid lines. For those charge states where more than one transition was measured the average difference is shown. The differences between experiment and MCDF values are higher by about $2 \mathrm{eV}$ for transitions involving $2 s$ orbitals because of inaccuracies in calculating the QED terms. The difference with MBPT calculations for selected $2 s_{1 / 2}-2 p_{3 / 2}$ transitions are shown as solid and open squares corresponding to two different starting potentials used in the calculations. 

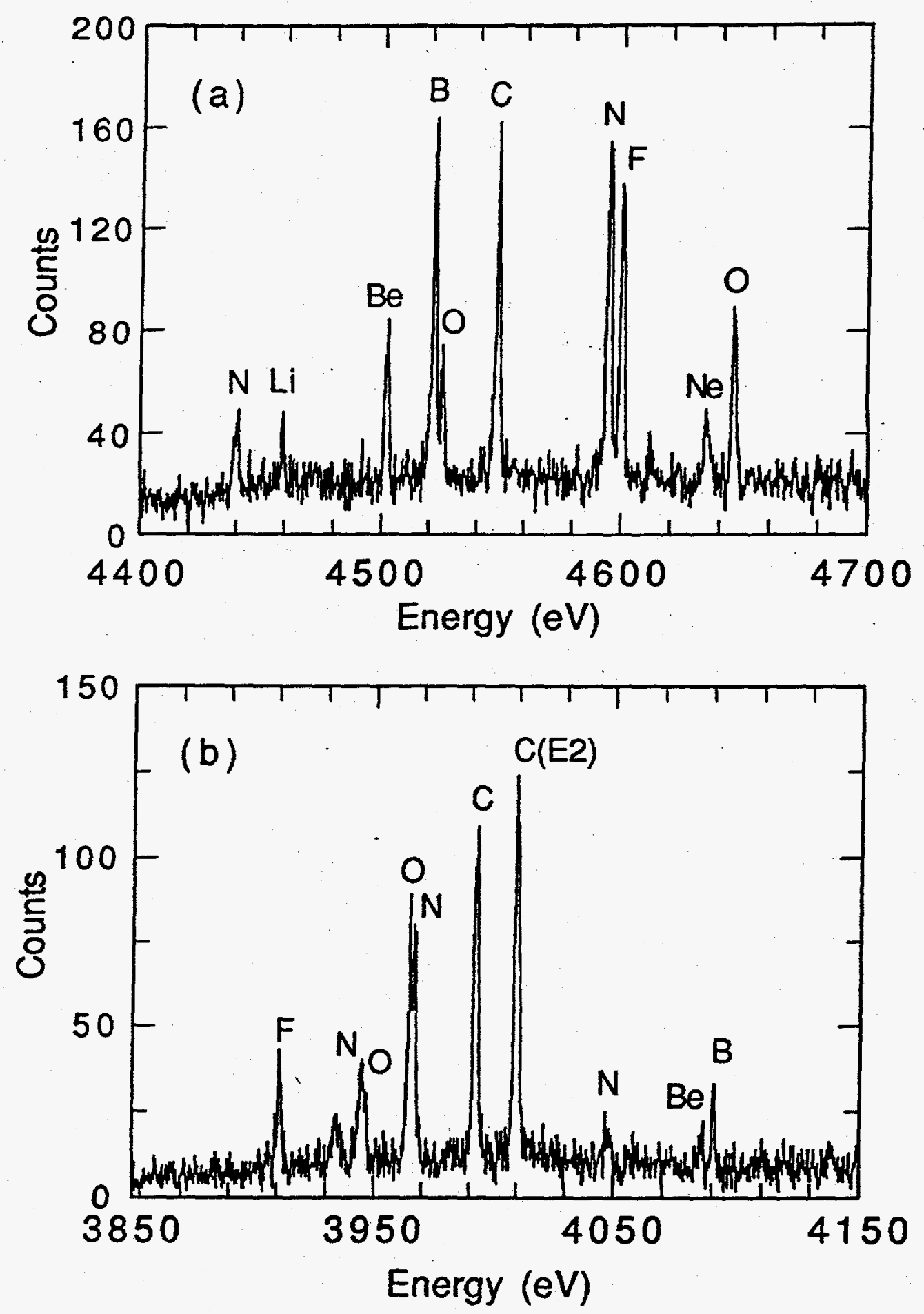

Figure 1. 


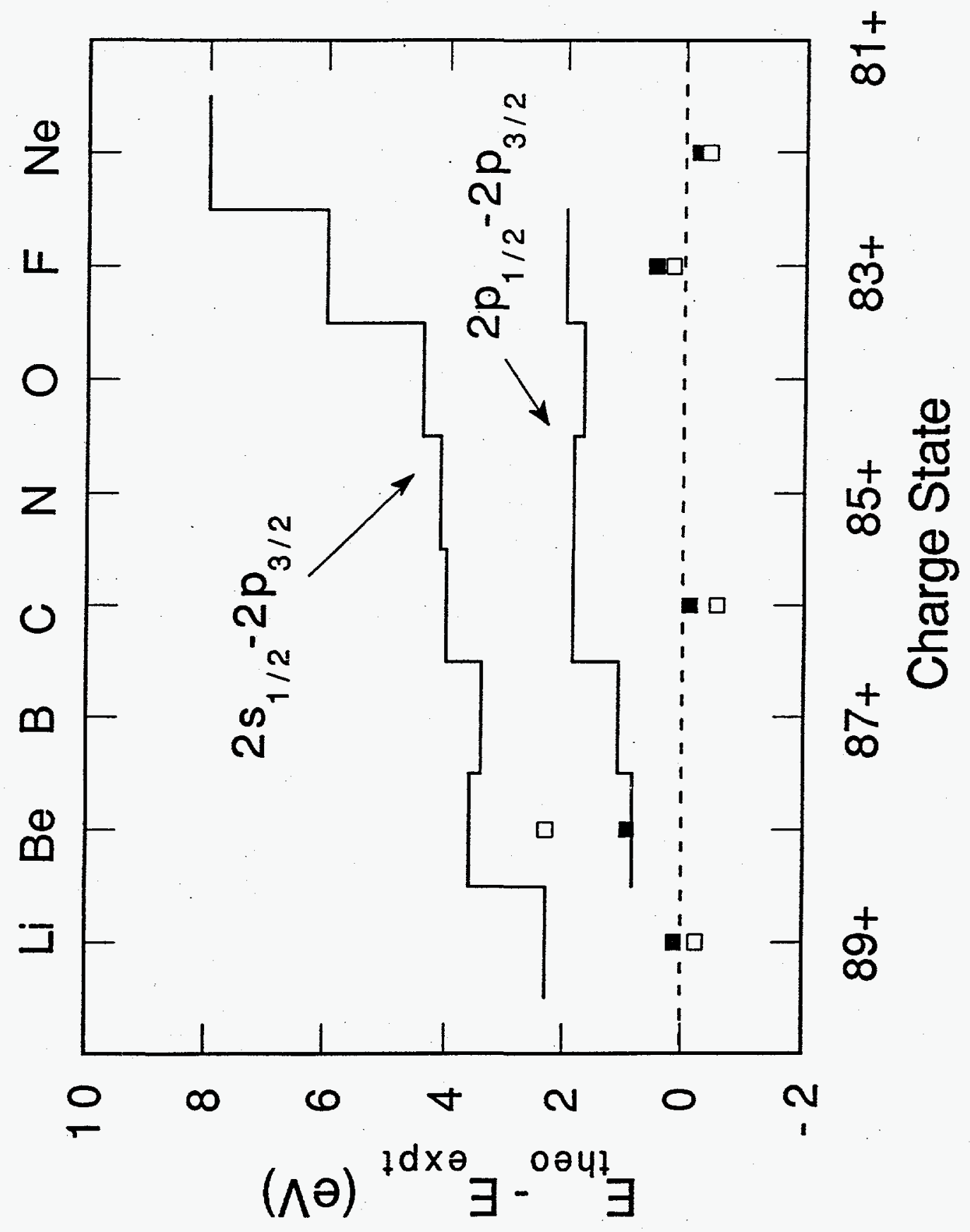

㐫 